\title{
Immunomodulatory synergy by combination of atorvastatin and glatiramer acetate in treatment of CNS autoimmunity
}

\author{
Olaf Stüve, ${ }^{1,2,3}$ Sawsan Youssef, ${ }^{4}$ Martin S. Weber, ${ }^{1}$ Stefan Nessler, ${ }^{2}$ Hans-Christian von Büdingen, ${ }^{5}$ \\ Bernhard Hemmer, ${ }^{2}$ Thomas Prod'homme, ${ }^{1}$ Raymond A. Sobel, ${ }^{6}$ Lawrence Steinman, ${ }^{4}$ \\ and Scott S. Zamvil'

\begin{abstract}
1Department of Neurology and Program in Immunology, University of California, San Francisco, San Francisco, California, USA. 'Department of Neurology, Heinrich Heine University, Dusseldorf, Germany. ${ }^{3}$ Neurology Section, VA North Texas Health Care System, Medical Service, Dallas, Texas, USA. ${ }^{4}$ Department of Neurology and Neurological Sciences, Interdepartmental Program in Immunology, Stanford University, Stanford, California, USA. ${ }^{5}$ Department of Neurology, University Hospital Zürich, Zurich, Switzerland. ${ }^{6}$ Department of Pathology, Stanford University, Stanford, California, USA.
\end{abstract}

\begin{abstract}
One approach to improving efficacy in MS therapy is to identify medications that provide additive or synergistic benefit in combination. Orally administered cholesterol-lowering HMG-CoA reductase inhibitors (known as statins), which exhibit immunomodulatory properties and are effective in treatment of the MS model EAE, are being tested in MS. As atorvastatin can enhance protective Th2 responses and has a different mechanism of action than glatiramer acetate (GA), a parenterally administered immunomodulatory agent approved for MS treatment, we tested whether the combination of these agents could be beneficial in EAE. Combination therapy using suboptimal doses of atorvastatin and GA prevented or reversed clinical and histologic EAE. Secretion of proinflammatory Th1 cytokines was reduced - and conversely Th 2 cytokine secretion was increased - in these mice, but not in mice treated with each drug alone at the same doses. Monocytes treated with the combination of suboptimal doses of atorvastatin and GA secreted an antiinflammatory type II cytokine pattern and, when used as APCs, promoted Th2 differentiation of naive myelin-specific $T$ cells. Our results demonstrate that agents with different mechanisms of immune modulation can combine in a synergistic manner for the treatment of CNS autoimmunity and provide rationale for testing the combination of atorvastatin and GA in MS.
\end{abstract}

\section{Introduction}

MS is an inflammatory autoimmune CNS demyelinating disease that is thought to be mediated in part by myelin-specific lymphocytes (1-3). Different classes of immunomodulatory agents with distinct mechanisms of action are approved for MS treatment (4-6). However, the current MS medications are only partially effective; they can be associated with side effects and potential toxicities, and there is ongoing debate regarding long-term efficacy of certain agents $(7,8)$. While one strategy to improve MS therapy is to develop novel agents that may have greater efficacy, it is important to identify existing or novel classes of drugs that may complement one another in combination to provide additive or synergistic benefit (9).

Glatiramer acetate (GA, also referred to as Copaxone and copolymer 1) is an immunomodulatory agent approved for treatment of relapsing-remitting MS (5). GA is a synthetic basic random copolymer composed of tyrosine $(\mathrm{Y})$, glutamate $(\mathrm{E})$, alanine $(\mathrm{A})$, and lysine $(\mathrm{K})$ that appears to preferentially affect $\mathrm{T}$ cells specific for CNS autoantigens (10), altering their antigen/MHC recognition in a manner similar to that of altered peptide ligands (11). Sustained treatment with GA in MS patients has been associated with the secretion of protective Th2 cytokines by some myelin-reactive $\mathrm{CD}^{+} \mathrm{T}$ cells $(12,13)$. Recent data obtained from GA-treated MS patients suggest that GA also mediates immunomodula-

Nonstandard abbreviations used: GA, glatiramer acetate; IFA, incomplete Freund's adjuvant; MBP, myelin basic protein.

Conflict of interest: The authors have declared that no conflict of interest exists. Citation for this article: J. Clin. Invest. 116:1037-1044 (2006). doi:10.1172/JCI25805. tory activity on APCs, promoting secretion of antiinflammatory cytokines and inhibiting secretion of proinflammatory cytokines (14-17). One can envisage that an agent that augments GA-mediated immunomodulation of myelin-reactive lymphocytes or APCs could enhance the efficacy of GA in MS therapy $(9,18)$.

Recent studies have demonstrated that oral cholesterol-lowering HMG-CoA reductase inhibitors (known as statins) have immunomodulatory properties that may be beneficial in the treatment of $\mathrm{T}$ cell-mediated, organ-specific autoimmune diseases and other inflammatory conditions (19-21). Promising results were obtained in initial clinical trials testing simvastatin (Zocor) and atorvastatin (Lipitor) in MS (22) and RA (23), respectively. Atorvastatin is currently being tested in a placebo-controlled trial in early MS (http:// immunetolerance.org/staycis/). In EAE models, atorvastatin has been shown to promote differentiation and expansion of myelin protein-reactive regulatory Th2 cells and to suppress upregulation of MHC class II and costimulatory molecules on APCs, indicating that the beneficial immunomodulatory effects of statins may involve both APC and T cell compartments $(24,25)$. Mevalonate, the product of HMG-CoA reductase, can reverse most, if not all, statin-induced immune effects on $\operatorname{APCs}(24,26)$ and T cells $(24,25$, $27)$, indicating that statins mediate immunomodulation by interfering with synthesis of mevalonate and its isoprenoid metabolites that are involved in posttranslational modification of GTP-binding signaling molecules. As atorvastatin treatment can promote the development of protective myelin-reactive Th 2 cells and does so utilizing a different mechanism of action than GA, we have tested whether atorvastatin could augment the therapeutic and immunomodulatory effects of GA on myelin-reactive T cells in EAE. 

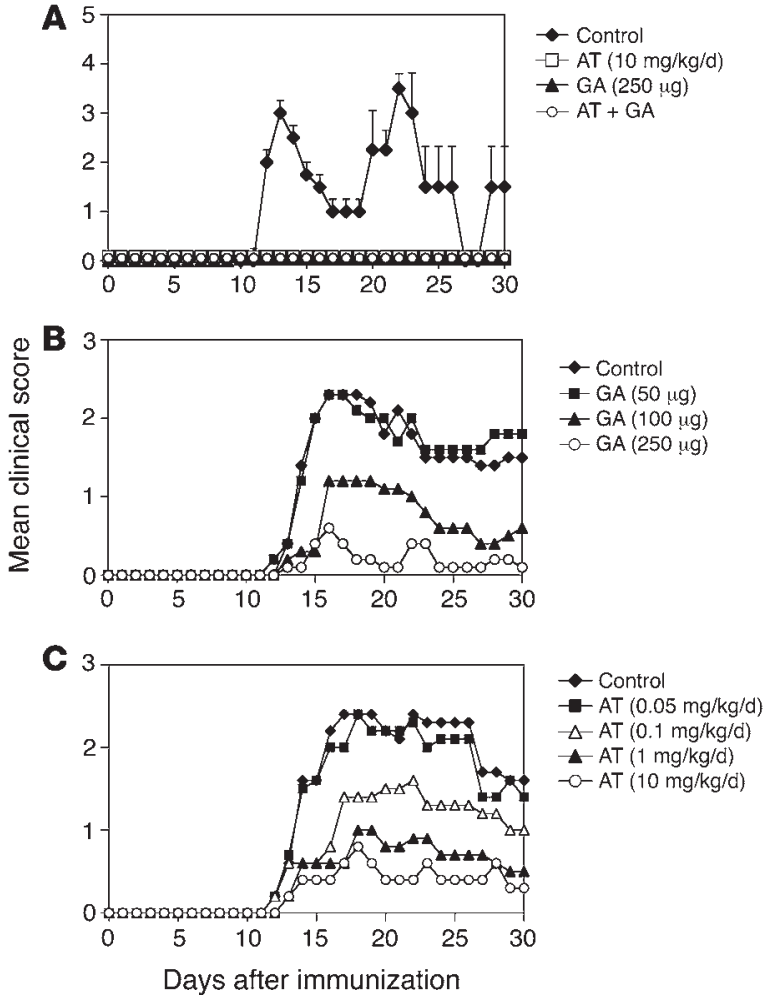

In this report we demonstrate that atorvastatin and GA can complement each other in a synergistic manner in EAE treatment. Clinical EAE was prevented or reversed in mice by combination therapy using suboptimal doses of atorvastatin and GA and was associated with reduced CNS inflammation and less demyelination than in mice treated with either drug alone at the same doses. This combination therapy was associated with enhanced secretion of protective Th 2 cytokines and reduced production of proinflammatory Th1 cytokines. Monocytes treated with this combination secreted a type II antiinflammatory cytokine pattern and promoted Th2 differentiation of naive myelin-specific T cells, suggesting that 1 mechanism that contributed to the development of this clinical and immunomodulatory synergy occurred at the level of the APC. Our results highlight how the EAE model can be used in preclinical screening to identify complementary activity between agents that might be considered for combination therapy in MS.

\section{Results}

Atorvastatin and GA in combination do not antagonize each other. While it is considered advantageous to combine medications with complementary activities in MS therapy, there is also concern that certain immunomodulatory agents could antagonize one another (28). In order to ensure that there was no unforeseen antagonism, we first tested atorvastatin and GA in combination using individual therapeutic doses. In a representative experiment, shown in Figure 1A, we observed that oral administration of $10 \mathrm{mg} / \mathrm{kg} / \mathrm{d}$ atorvastatin or a single s.c. injection of $250 \mu \mathrm{g}$ GA in incomplete Freund's adjuvant (IFA) alone prevented myelin basic protein $(\mathrm{MBP})$ peptide Ac1-11-induced EAE in $(\mathrm{PL} / \mathrm{J} \times \mathrm{SJL} / \mathrm{J}) \mathrm{F}_{1}$ mice. When atorvastatin and GA were administered together using these same optimal doses, this combination was equally effective as the individual agents alone. Thus there was no evident antagonism.

\section{Figure 1}

Atorvastatin and GA in combination do not antagonize each other. (A) Therapeutic doses of oral atorvastatin (AT; $10 \mathrm{mg} / \mathrm{kg} / \mathrm{d}$ ) and GA (250 $\mu \mathrm{g}$ in IFA) were administered in combination to $(\mathrm{PL} / \mathrm{J} \times \mathrm{SJL} / \mathrm{J}) \mathrm{F}_{1}$ mice (10 per group) immunized with MBP Ac1-11 (100 $\mu \mathrm{g})$ in CFA. GA was administered once (s.c. in IFA) 7 days prior to immunization (day -7). Daily atorvastatin $(0.05 \mathrm{mg} / \mathrm{kg} / \mathrm{d})$ treatment began 2 days prior to $\mathrm{GA}$ injection (day -9). Control mice received daily oral PBS (vehicle) and 1 s.c. injection of IFA. (B) Dose titration to identify a suboptimal GA dose in prevention of EAE. GA $(50,100$, or $250 \mu \mathrm{g}$ in IFA) was administered s.c. on day -7 ( 5 mice per group). Control mice received a single s.c. injection of IFA. (C) Dose titration to identify a suboptimal atorvastatin dose in prevention of EAE. Atorvastatin $(0.05,0.1,1$, or $10 \mathrm{mg} / \mathrm{kg} / \mathrm{d})$ was administered daily by oral gavage to mice (5 per group) starting 9 days prior to immunization with MBP Ac1-11. Control mice received daily oral PBS. Data are representative of 3 separate experiments.

We also did not observe antagonism when we tested a lower therapeutic dose $(1 \mathrm{mg} / \mathrm{kg} / \mathrm{d})$ of atorvastatin in combination with 250 $\mu \mathrm{g}$ GA (data not shown).

Combination therapy using suboptimal doses of atorvastatin and GA prevents clinical and histologic EAE. In order to evaluate combination therapy in the EAE model using medications that are individually fully effective, it is necessary to test these drugs in combination using suboptimal doses (9). In dose-response experiments we consistently observed that $50 \mu \mathrm{g}$ GA in IFA administered prior to EAE induction was no more effective than control (vehicle only) treatment (Figure 1B and Table 1). Similarly, we observed that daily oral treatment of atorvastatin $(0.05 \mathrm{mg} / \mathrm{kg} / \mathrm{d})$ begun prior to EAE induction had no detectable effect (Figure 1C and Table 1). Thus, in order to test whether these 2 drugs could complement one another, we combined $0.05 \mathrm{mg} / \mathrm{kg} / \mathrm{d}$ atorvastatin with $50 \mu \mathrm{g}$ GA. The combination of both medications at those individually suboptimal doses prevented clinical signs of EAE (Figure 2A and Table 1). In fact, this combination was as effective as treatment with each individual agent at its optimal dose (atorvastatin, $10 \mathrm{mg} / \mathrm{kg} / \mathrm{d} ; \mathrm{GA}$, $250 \mu$; compare Figure 2A with Figure 1, B and C).

Mice treated with suboptimal doses of atorvastatin or GA alone or in combination were evaluated for histologic EAE. There were no detectable differences in the numbers of CNS inflammatory foci in untreated mice with EAE and mice treated with subopti-

\section{Table 1}

Combination of atorvastatin and GA suppresses clinical and histologic EAE

\begin{tabular}{lccc}
$\begin{array}{l}\text { Treatment } \\
\text { group }\end{array}$ & $\begin{array}{c}\text { Disease } \\
\text { incidence }^{A}\end{array}$ & $\begin{array}{c}\text { Mean disease } \\
\text { severity (SEM) }\end{array}$ & $\begin{array}{c}\text { Mean total } \\
\text { inflammatory } \\
\text { foci (SEM) }\end{array}$ \\
$\begin{array}{l}\text { PBS } \\
\text { GA }(50 \mu \mathrm{g})^{\mathrm{C}}\end{array}$ & $10 / 10$ & $3.6(0.29)$ & $39(8)$ \\
$\begin{array}{l}\text { Atorvastatin } \\
(0.05 \mathrm{mg} / \mathrm{kg} / \mathrm{d})^{\mathrm{B}}\end{array}$ & $10 / 10$ & $3.5(0.56)$ & $48(6)$ \\
Combination & $3 / 10$ & $4(0)$ & $37(11)$ \\
& & $1.4(0.19)$ & $15(2)^{\mathrm{D}}$ \\
\hline
\end{tabular}

AEAE was induced by immunization of female $(P L / J \times S J L / J) F_{1}$ mice with $100 \mu \mathrm{g}$ MBP Ac1-11 s.c in CFA on day 0. BPBS and atorvastatin were administered once daily in a volume of $0.5 \mathrm{ml}$, utilizing $20 \mathrm{~mm}$ feeding needles, starting on day -9 . CMice received 1 s.c. injection with a volume of $0.1 \mathrm{ml}$ of an emulsion consisting of $50 \%$ IFA and $50 \% \mathrm{GA}$ suspended in PBS on day -7 . ${ }^{D} P<0.005$ by 1 -way ANOVA. 
A
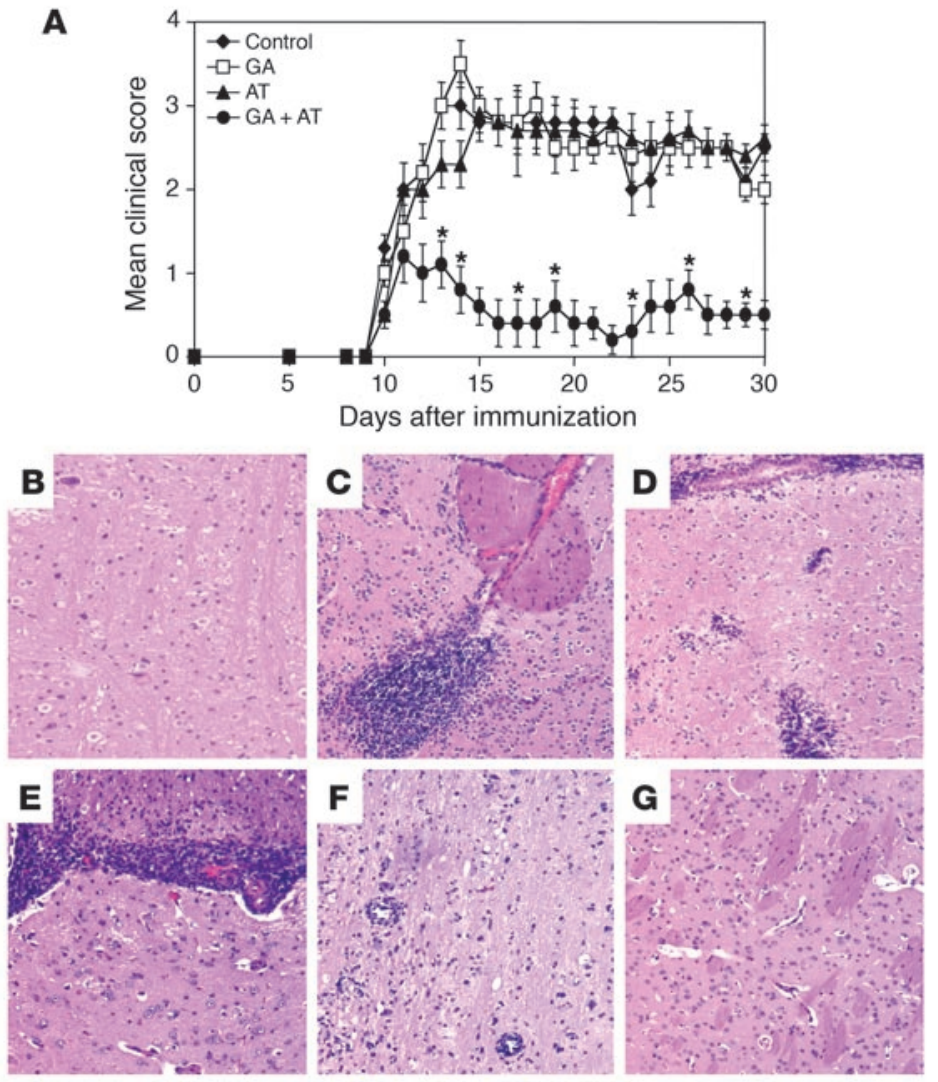

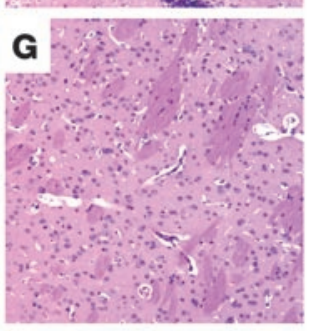

\section{Figure 2}

Combination therapy using suboptimal doses of atorvastatin and GA prevents clinical and histologic EAE. (A) On day 0, $(\mathrm{PL} / \mathrm{J} \times \mathrm{SJL} / \mathrm{J}) \mathrm{F}_{1}$ mice (10 per group) were immunized with MBP Ac1-11 (100 $\mu \mathrm{g})$. GA (50 $\mu \mathrm{g}$ in IFA) was administered once on day -7 . Daily atorvastatin $(0.05 \mathrm{mg} / \mathrm{kg} / \mathrm{d})$ was begun 2 days prior to GA injection (day -9 ). Control mice received daily oral PBS (vehicle) starting 2 days prior to a s.c. injection of IFA on day -7 . Shown are group scores \pm SEM. Data are representative of 4 separate experiments. Comparisons were made between the group treated with combination therapy and the groups that received either atorvastatin treatment alone, GA treatment alone, or no treatment. ${ }^{*} P<0.005$ by ANOVA, which was considered significant. (B-G) Combination of atorvastatin and GA suppresses histologic EAE in brain tissue. (B) No infiltrates were detected in unimmunized $(P L / J \times S J L / J) F_{1}$ mice. (C-E) EAE lesions with infiltrates of mononuclear cells were detected in vehicle-treated MBP Ac1-11-immunized mice (C) and mice treated with suboptimal doses of oral atorvastatin ( $0.05 \mathrm{mg} / \mathrm{kg} / \mathrm{d}$; D) or GA (50 $\mu \mathrm{g}$; E). (F and G) Reduced CNS infiltration was observed in mice treated with the combination of suboptimal doses of atorvastatin and GA. CNS tissue was harvested, fixed, and stained with H\&E (magnification, $\times 40$ ) as described in Methods. mal doses of either oral atorvastatin or GA (Table 1). In contrast, the number of CNS inflammatory infiltrates was significantly reduced in mice treated with the combination of suboptimal doses of these agents (Table 1). Representative histologic figures from the brains of representative mice from each treatment group are shown in Figure 2, B-G.

Combination therapy using suboptimal doses of atorvastatin and GA reverses clinical and histologic EAE. In general, immunomodulatory therapy in MS is initiated after patients have developed clinical signs or symptoms of CNS demyelinating disease. Therefore, it is important to test whether a novel treatment regimen, which is effective EAE prevention, can also reverse established disease. We evaluated whether suboptimal doses of atorvastatin in combination could reverse established EAE. Typically in EAE studies GA is administered in IFA once prior to disease induction (29-31). In order to test GA in EAE reversal, we established a treatment protocol administering GA s.c. daily in aqueous solution (PBS) in a manner similar to its use in the treatment of MS patients. Treatment was initiated when individual mice developed a clinical score of $\geq 2$ (see Methods). We consistently observed that daily treatment with either $50 \mu \mathrm{g}$ GA s.c. or $0.05 \mathrm{mg} / \mathrm{kg} / \mathrm{d}$ oral atorvastatin after EAE onset had no detectable effect on disease progression (Figure $3 \mathrm{~A})$. However, treatment using the combination of these suboptimal doses of atorvastatin and GA reversed clinical severity of EAE (Figure 3A). Further, clinical improvement by treatment with this combination was associated with reduced CNS inflammation (Figure 3, B-E) and less demyelination (Figure 3E). Fewer swollen axons were observed in the combined treatment group (Figure 3E), suggesting that there may be a decrease in axonal injury.
Combination of atorvastatin and GA promotes induction of Th2 myelinreactive T cells. GA treatment in $\operatorname{MS}(12,13)$ and $\operatorname{EAE}(30,31)$ has been associated with the induction of Th2 myelin-reactive T cells. Presumably acting through a different mechanism of action, oral atorvastatin treatment in EAE also promotes the development of myelin-reactive Th 2 cells. Thus, we tested whether the synergistic beneficial effects observed using the combination of atorvastatin and GA was associated with secretion of Th2 cytokines. In vivo administration of suboptimal doses of atorvastatin and GA in combination markedly suppressed the secretion of Th1 cytokines IFN- $\gamma$, IL-12, and TNF- $\alpha$ by MBP-reactive T cells (Figure 4). Conversely, secretion of Th 2 cytokines IL- 4 and IL- 10 by MBP-reactive $\mathrm{T}$ cells was enhanced in mice treated with this combination. In contrast, lymphocytes from mice treated individually with atorvastatin or GA secreted predominantly Th1 cytokines in a manner similar to control mice.

Combination of atorvastatin and GA can promote Th2 differentiation through immunomodulation of monocyte APCs. It is recognized that GA may have direct and indirect effects on APCs, which could contribute to its immunomodulatory effects in MS treatment (14-16). GA treatment of monocytes/macrophages $(15,16)$ and dendritic cells (14) promotes their secretion of antiinflammatory type II cytokines (16), which presumably contributes to the differentiation of GA-reactive T cells into Th2 cells. Data indicate that statins also have antiinflammatory effects on APC subpopulations, including monocytes/macrophages $(24,26)$. As we observed that the combination of atorvastatin and GA was beneficial in EAE and synergistically promoted secretion of Th2 cytokines, we tested whether atorvastatin and GA in combination might alter 

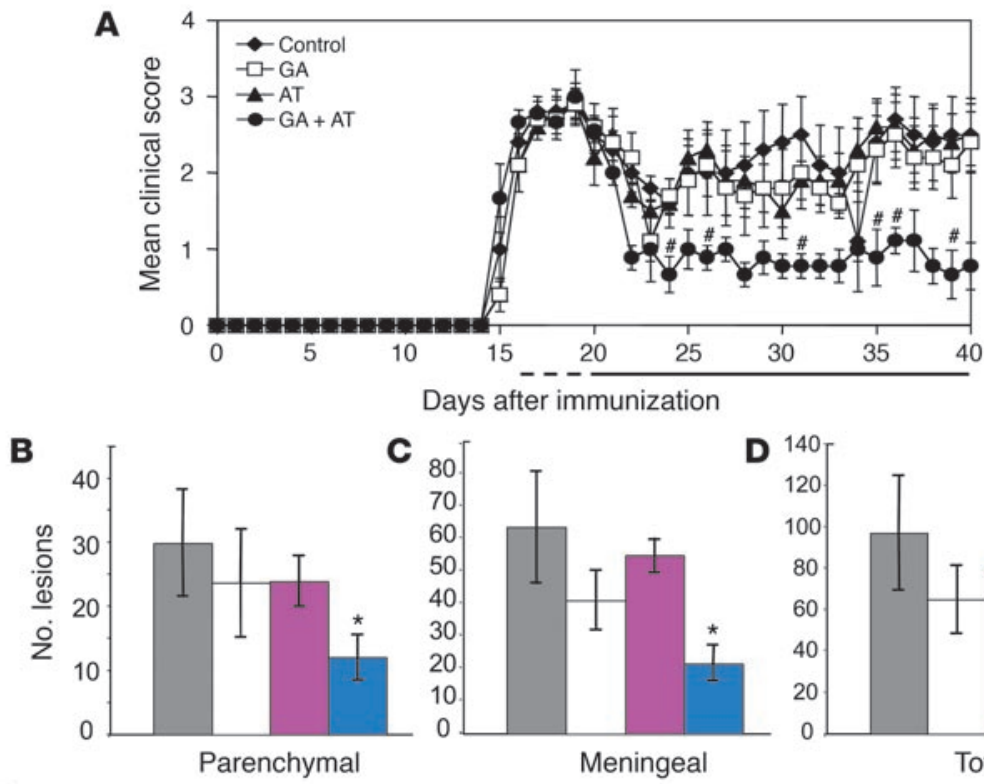

E
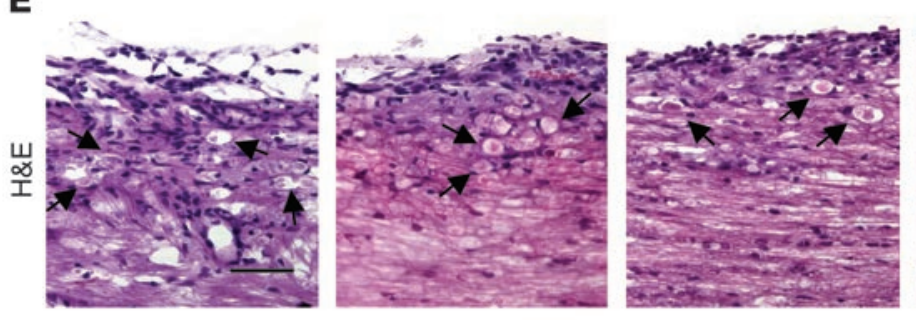

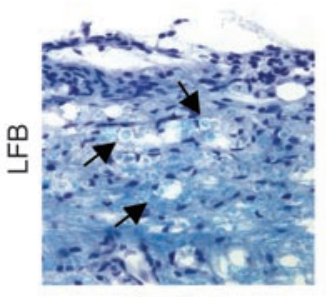

Control

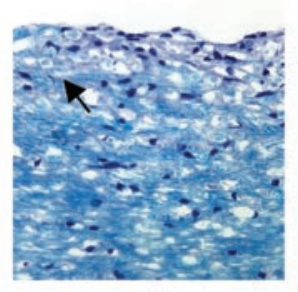

AT

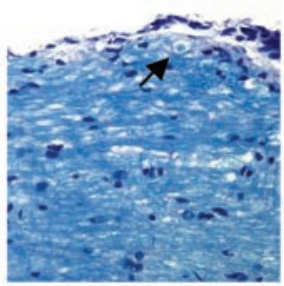

GA

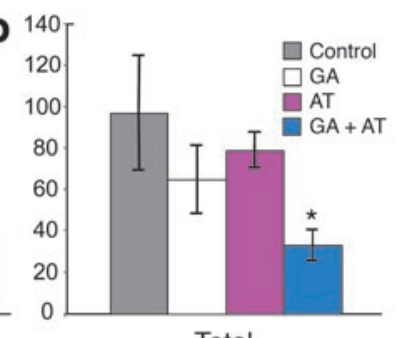

Total
Figure 3

Combination therapy using suboptimal doses of atorvastatin and GA reverses clinical and histologic EAE. (A) On day 0, (PL/J $\times$ SJL/J)F $F_{1}$ mice were immunized with MBP Ac1-11 (100 $\left.\mu \mathrm{g}\right)$. Mice were randomized (10 per group) to start treatment when they developed a clinical disease score $\geq 2$ (dashed line; solid line indicates continued treatment after all mice were randomized). Treatment groups included suboptimal doses of GA ( $50 \mu \mathrm{g} / \mathrm{d}$ s.c. and PBS orally), atorvastatin $(0.05 \mathrm{mg} / \mathrm{kg} / \mathrm{d}$ orally and PBS s.c.) and combination of these 2 medications. Control mice received PBS (vehicle) s.c. and orally. Shown are group scores \pm SEM. Data shown are representative of 2 separate experiments. Comparisons were made between the group treated with combination therapy and the groups that received either atorvastatin treatment alone, GA treatment alone, or no treatment. ${ }^{\#} P<0.001$ by Mann-Whitney $U$ test, which was considered significant. (B-D) Histologic analysis revealed a reduced number of parenchymal (B), meningeal (C), and total (D) inflammatory foci in mice treated with the combination of suboptimal doses of atorvastatin and $\mathrm{GA}$. ${ }^{*} P<0.008$ by 1 -way, multiple-range ANOVA for multiple comparisons. (E) Mice treated with the combination of suboptimal doses of atorvastatin and GA showed reduced inflammatory cell infiltration (upper panels, H\&E), demyelination (lower panels, Luxol fast blue-hematoxylin [LFB]), and axonal injury (arrows indicate swollen axons). Original magnification, $\times 160$. Scale bar: $50 \mu \mathrm{m}$.

the proinflammatory and antiinflammatory cytokine secretion profile of APCs in an additive or synergistic manner. As shown in Figure 5, treatment of monocytes with either atorvastatin or GA at higher concentrations suppressed secretion of proinflamma-

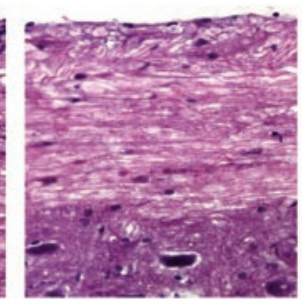

tory cytokines IL-12 and TNF- $\alpha$ and promoted secretion of the antiinflammatory cytokine IL-10. When titrated to suboptimal concentrations, neither atorvastatin nor GA affected monocyte secretion of these cytokines. In contrast, combination therapy using suboptimal doses of both agents suppressed monocyte secretion of TNF- $\alpha$ and IL- 12 while promoting IL-10 secretion (Figure 5). Furthermore, monocytes that had been pretreated with suboptimal doses of atorvastatin and GA in combination, promoted Th2 differentiation of naive myelin-specific T cells (Figure 6). These data demonstrate that in combination these 2 drugs can alter the secretion of APC-derived T cell polarizing cytokines, enhancing myelin-specific Th2 responses.

\section{Discussion}

Several criteria should be considered when selecting immunomodulatory agents for use in combination therapy for MS: (a) medications should have complementary activities, preferentially acting through different mechanisms of action; (b) ideally each medication should have an excellent safety profile; and (c) the combination should not create additional toxicities. In this regard, the 2 FDA-approved medications used in this investigation are well tolerated and have no apparent overlapping toxicities $(5,32)$. Data from previous studies indicate that atorvastatin mediates immunomodulatory effects on $\mathrm{T}$ cells and APCs primarily through inhibition of synthesis of isoprenoid compounds in the mevalonate pathway $(24,25,27)$. Isoprenylation of small GTP-binding proteins is necessary for their intracellular trafficking and subcellular localization to the cytoplasmic surface of the plasma membrane (33). Possibly by reducing available ras, which has an important role in ERK activation and Th1 differentiation (34), statins may facilitate Th2 differentiation (35). In contrast, GA is considered an antigen-based immunomodulatory agent that has been associated with enhanced secretion of antiinflammatory cytokines by myelin-reactive T cells $(12,13)$ and APCs (14-16). Thus the mechanisms of action of these 2 drugs are distinct. Having met all of the criteria described above and having demonstrated that optimal doses of atorvastatin and GA did not antagonize one another, we reasoned that these 2 drugs were excellent candidates to be tested in combination therapy. 

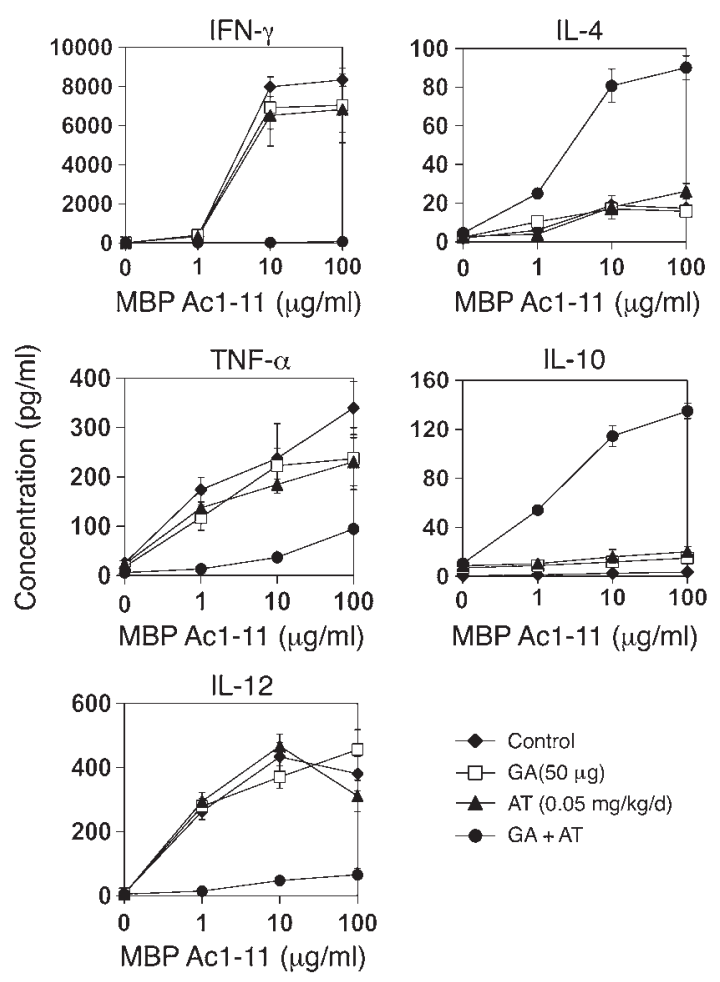

In this report we established that atorvastatin and GA had synergistic clinical and immunological effects. The combination of suboptimal doses of GA and atorvastatin was as effective in EAE prevention as the optimal dose of either drug alone and promoted Th2 immunomodulation. More importantly, the combination of suboptimal doses of atorvastatin and GA, administered s.c. in aqueous solution, effectively reversed paralysis when daily treatment was initiated after EAE was established. Further, treatment of EAE with this combination was associated with a reduced number of CNS inflammatory lesions and less demyelination. In order to investigate one potential mechanism responsible for the Th2 bias, we examined whether atorvastatin and GA have a combined immunomodulatory effect on APCs. Our results indicate that the combination of GA and atorvastatin facilitated the differentiation of type II (16) monocytes that secreted an antiinflammatory profile of $\mathrm{T}$ cell-polarizing cytokines. Indeed, monocytes

\section{Figure 5}

Combination therapy of atorvastatin and GA reduces secretion of proinflammatory cytokines and promotes secretion of IL-10 by monocytes. Bone marrow-derived monocytes/macrophages were isolated from $(\mathrm{PL} / \mathrm{J} \times \mathrm{SJL} / \mathrm{J}) \mathrm{F}_{1}$ mice as described in Methods. Treatment of monocytes with optimal doses of atorvastatin $(10 \mu \mathrm{M})$ or GA $(50 \mu \mathrm{M})$ suppressed secretion of proinflammatory cytokines IL-12 and TNF- $\alpha$ and promoted secretion of the antiinflammatory cytokine IL-10. Suboptimal doses of atorvastatin $(0.1 \mu \mathrm{M})$ or $\mathrm{GA}$ alone $(6.25 \mu \mathrm{M})$ did not alter cytokine secretion, whereas treatment using the combination of these suboptimal doses suppressed secretion of TNF- $\alpha$ and IL-12 and promoted IL-10 secretion. Monocytes in each group were pretreated with IFN- $\gamma$ for 24 hours. Cytokine secretion by unstimulated monocytes was measured and recorded: IL-12, $112( \pm 2)$; TNF- $\alpha, 153( \pm 2)$; and $\mathrm{IL}-10,141( \pm 3) \mathrm{pg} / \mathrm{ml}$. Treatment groups were compared to the control group (untreated) using 1-way ANOVA. Data are representative of 3 separate experiments. ${ }^{*} P<0.05$.

\section{Figure 4}

The combination of atorvastatin and GA promotes secretion of antiinflammatory Th2 cytokines. On day $0,(\mathrm{PL} / \mathrm{J} \times \mathrm{SJL} / \mathrm{J}) \mathrm{F}_{1}$ mice were immunized with MBP Ac1-11. GA $(50 \mu \mathrm{g})$ was administered s.c. on day -7 . Daily atorvastatin $(0.05 \mathrm{mg} / \mathrm{kg} / \mathrm{d})$ was begun 2 days prior to GA injection (day -9). Control mice received daily oral PBS (vehicle) and a s.c. injection of IFA only. Spleen cells were obtained on day 10 and cultured $(5 \times 105 /$ well $)$ in the presence of MBP Ac1-11 at the doses indicated. Supernatants were examined for Th1 (IFN- $\gamma$, TNF- $\alpha$, and IL-12) and Th2 (IL-4 and IL-10) cytokines by ELISA as described in Methods. Mean \pm SEM are shown. Data are representative of 3 separate experiments.

matured in the presence of the combination of atorvastatin and GA promoted Th2 differentiation of naive myelin-specific T cells. While other mechanisms may contribute, we have established one potential mechanism that could account for the observed clinical and immunological synergistic effects of this combination. Our results clearly support testing the combination of atorvastatin and GA in clinical MS trials.

Other approved immunomodulatory agents are also being considered for combination therapy in MS (36). For example, the combination of GA and Avonex, a preparation of interferon $\beta$ - 1 a approved for MS treatment, was assessed for safety in a small trial of patients with relapsing-remitting MS (37) and is now being tested for efficacy in a larger trial (http://www.combirx. org/). Although interferon $\beta$ acts through a different pathway than statins, these 2 drugs may overlap in their immunomodulatory effects. For example, both interferon $\beta$ and atorvastatin can induce secretion of antiinflammatory cytokines $(20,24,25$, $38,39)$. Like statins $(19,24)$, interferon $\beta$ also inhibits MHC class II upregulation on APCs (40). While statins inhibit transcription of the MHC class II transactivator (CIITA) $(19,24)$, the "master regulator" for MHC class II expression, interferon $\beta$ reduces CIITA activity (40). It is known that interferon $\beta$ is effective in reducing the number of new radiographically detectable inflammatory MS lesions $(41,42)$, presumably by inhibiting secretion of proteases
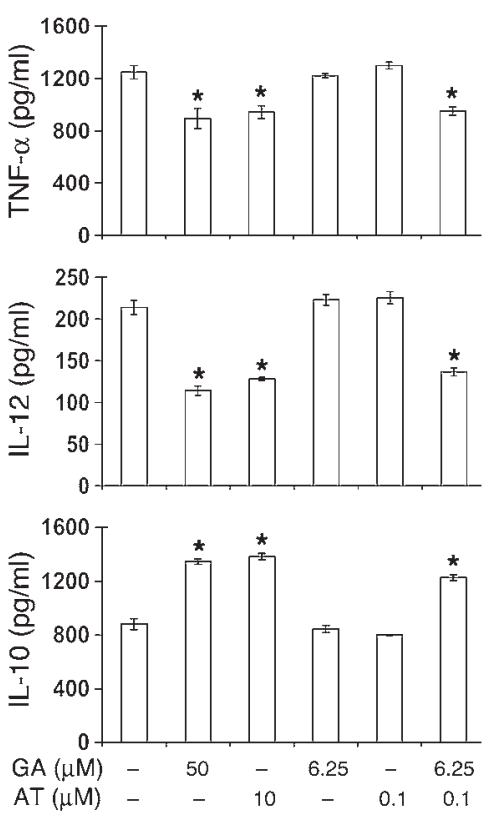

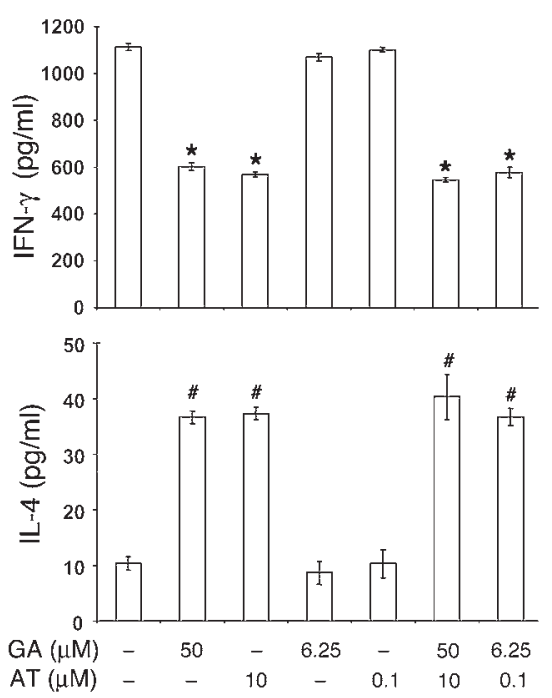

and preventing upregulation of adhesion/costimulatory molecules that participate in leukocyte trafficking into the CNS (43, 44). Data indicate that through inhibition of rho prenylation in endothelial cells (45), statin treatment may also inhibit leukocyte trafficking into the CNS $(22,27)$. Since it is unclear whether the pleiotropic activities of these 2 drugs will be complementary, studies are necessary to evaluate whether the combination of interferon $\beta$ and statins will be antagonistic or beneficial (46).

As we have shown in this investigation, the EAE model is useful for initial testing of potential combination therapies for MS. One must consider that FDA-approved therapies and several novel therapies, administered individually, potently suppress EAE. As we have described, one strategy to evaluate potential complementary therapeutic benefit is to administer candidate drugs using suboptimal doses. In this regard, atorvastatin and GA were synergistic when administered at doses that had no detectable clinical or immunomodulatory effects alone. The general principles that we have established in this study should be applicable for evaluating other combinations of immunomodulatory agents in the EAE model.

\section{Methods}

Mice. Female $(\mathrm{PL} / \mathrm{J} \times \mathrm{SJL} / \mathrm{J}) \mathrm{F}_{1}$ mice, 5-8 weeks of age, were purchased from The Jackson Laboratory. B10.PL MBP Ac1-11-specific TCR Tg mice (47) were kindly provided by V. Kuchroo (Harvard University, Cambridge, Massachusetts, USA). All animal protocols were approved by the Committee on Animal Research at the University of California, San Francisco, and the Division of Comparative Medicine at Stanford University, in accordance with NIH guidelines.

Peptide. Mouse MBP peptide Ac1-11 (ASQKRPSQRHG) was synthesized by solid-phase Fmoc chemistry by Quality Control Biochemicals Inc. After cleavage from the solid support and deprotection of the amino acid side chains, peptides were purified by reverse-phase HPLC (C18 column; YMC). Major peaks, analyzed by MALDI-TOF mass spectrometry and HPLC, contained greater than $95 \%$ of the desired product.

EAE induction and clinical evaluation. Female $(\mathrm{PL} / \mathrm{J} \times \mathrm{SJL} / \mathrm{J}) \mathrm{F}_{1}$ mice, 8-12 weeks old, received a s.c. injection in the lower flanks of $100 \mu \mathrm{g}$ MBP Ac1-11 in $0.1 \mathrm{ml}$ PBS emulsified in an equal volume of CFA supplemented with 2 $\mathrm{mg} / \mathrm{ml}$ of mycobacterium tuberculosis H37Ra (DIFCO Laboratories) on day 0 . Immediately thereafter, and again 48 hours later, mice received an i.v. injection of $400 \mathrm{ng}$ pertussis toxin in $0.2 \mathrm{ml}$ PBS. Individual animals were

\section{Figure 6}

Monocytes pretreated with the combination of suboptimal doses of atorvastatin and GA promote Th2 differentiation. Bone marrow-derived monocytes/macrophages isolated from $(\mathrm{PL} / \mathrm{J} \times \mathrm{SJL} / \mathrm{J}) \mathrm{F}_{1}$ mice were treated with optimal and suboptimal doses of atorvastatin (10 and 0.1 $\mu \mathrm{M}$, respectively) or $\mathrm{GA}$ (50 and $6.25 \mu \mathrm{M}$, respectively) alone or in combination. Pretreated monocytes were washed and cocultured with naive myelin-specific T cells from B10.PL MBP TCR Tg mice in the presence of MBP Ac1-11. Th1 and Th2 differentiation was evaluated by measurement of IFN- $\gamma$ and IL-4, respectively. Treatment groups were compared with the control group using 1-way ANOVA. Data are representative of 2 separate experiments. ${ }^{\#} P<0.001$; ${ }^{*} P<0.05$. observed daily, and clinical scores were assessed in a blinded fashion on a $0-5$ scale: 0 , no clinical disease; 1 , loss of tail tone only; 2 , mild monoparesis or paraparesis; 3 , paraplegia; 4 , quadraparesis; and 5 , moribund or deceased.

Atorvastatin and GA treatments. Atorvastatin (prescription formulation; Pfizer) was brought into suspension in PBS as described previously (24). Atorvastatin $(0.05,0.1,1$, or $10 \mathrm{mg} / \mathrm{kg})$ was administered orally in 0.5 $\mathrm{ml}$ PBS once daily using a 20-mm feeding needle (Popper and Sons Inc.) starting on day -9, 2 days prior to GA administration (day -7). For EAE prevention, mice received 1 s.c. injection of a $0.1-\mathrm{ml}$ emulsion consisting of GA $(50,100$, or $250 \mu \mathrm{g})$ in an equal volume of PBS and IFA in the upper flanks. For EAE reversal, daily treatment with GA and atorvastatin began when a clinical score of $\geq 2.0$ was reached. GA $(50 \mu \mathrm{g} / \mathrm{d})$ was administered s.c. in $0.1 \mathrm{ml} \mathrm{PBS}$, and atorvastatin was administered by oral gavage. Purified atorvastatin used for in vitro studies was provided by R. Laskey (Pfizer Inc., New York, New York, USA). GA was provided by Teva Neuroscience.

Proliferation assays. For primary proliferative responses, $5 \times 10^{5}$ spleen cells removed from MBP Ac1-11-immunized mice were cultured in $0.2 \mathrm{ml}$ serum-free medium, X-Vivo 20 (BioWhittaker Inc.), supplemented with $5 \times 10^{-5} \mathrm{M} 2$-mercaptoethanol, $2 \mathrm{mM}$ glutamine, $100 \mu \mathrm{g} / \mathrm{ml}$ penicillin, and $100 \mu \mathrm{g} / \mathrm{ml}$ streptomycin. Primary splenocytes were cultured with appropriate concentrations of MBP Ac1-11 for 72 hours. CD4-purified naive MBP Ac1-11 TCR Tg T cells were cultured with purified monocytes for 48 hours. Cultures were pulsed with $1 \mu \mathrm{Ci}\left[{ }^{3} \mathrm{H}\right]$ thymidine and harvested 16 hours later. Mean cpm of $\left[{ }^{3} \mathrm{H}\right]$ thymidine incorporation was calculated for triplicate cultures. SD of triplicate cultures are shown.

Cytokine analysis. Cell culture supernatants were collected at 48-hour (IL-12 and TNF- $\alpha$ ), 72-hour (IFN- $\gamma$ ), and 120-hour (IL-4 and IL-10) incubation for cytokine analysis. Quantitative ELISA was performed using paired monoclonal Abs specific for corresponding cytokines per the manufacturer's recommendations (BD Biosciences - Pharmingen). The results of ELISA assays are expressed as an average of triplicate wells \pm SD. SOFTmax ELISA plate reader and software was used for data analysis (SoftMax Pro5; Molecular Devices Corp.).

Monocyte cultures. Bone marrow-derived monocytes were obtained from femurs of 10 -week-old $(\mathrm{PL} / \mathrm{J} \times \mathrm{SJL} / \mathrm{J}) \mathrm{F}_{1}$ mice. After flushing femurs with PBS, cells were passed through a $40-\mu \mathrm{m}$ strainer to obtain a single-cell suspension. Following centrifugation, bone marrow-derived cells were resuspended in L-929 conditioned medium containing macrophage colony-stimulating factor and GA or atorvastatin at the doses indicated and plated in 
24-well plates. After 5 days, monocyte cultures were counted and assessed for purity by FACS staining with anti-CD11b (BD Biosciences - Pharmingen) and F4/80 (CALTAG Laboratories). Purity was $>99 \%$. In order to test monocytes as APCs, monocyte cultures were washed, and $2 \times 10^{5}$ naive MBP Ac1-11-specific TCR Tg T cells were added in the presence of MBP Ac1-11.

Histopathology. Anesthetized mice were perfused with $20 \mathrm{ml}$ cold PBS. Brains and spinal cords were fixed in $4 \%$ paraformaldehyde and embedded in paraffin for the initial EAE treatment (prevention) experiments. For disease-reversal experiments, the tissues were immersed in $20 \%$ sucrose following fixation and frozen in OCT for cryosectioning. Sections were stained with H\&E or Luxol fast blue. Selected brain, thoracic, and lumbar spinal cord sections were evaluated by an examiner blinded to the treatment status of the animal. Inflammatory foci were counted in meninges and parenchyma.

Statistics. Unless otherwise indicated, data are presented as mean \pm SEM. For clinical scores, significance between groups was examined by MannWhitney $U$ test. All other statistical comparisons between groups were examined using 1-way multiple range ANOVA test for multiple comparisons. $P$ values less than 0.05 were considered statistically significant.

\section{Acknowledgments}

We thank R.P. Lisak, H.-P. Hartung, S.L. Hauser, J.W. Cohen Tervaert, and P.A. Nelson for helpful discussions. We thank R. Laskey for providing purified atorvastatin. S. Youssef is a fellow of the National Multiple Sclerosis Society (NMSS). M.S. Weber is supported by a fellowship from the Deutsche Forschungsgemeinschaft (DFG). Support was provided to S.S. Zamvil by grants from the NIH (RO1 AI05709), the NMSS (RG 3622-A), The Maisin Foundation, Teva Neuroscience, and The Dana Foundation; to L. Steinman by NIH grant RO1 AI05709 and NMSS grant RG 3622-A; to R.A. Sobel by NIH grant R01 NS 046414; and to B. Hemmer by DFG grants He2386/4-1 and He2386/4-2.

Received for publication May 31, 2005, and accepted in revised form January 24, 2006.

Address correspondence to: Scott S. Zamvil, Department of Neurology, University of California, San Francisco, 513 Parnassus Avenue, S-268, San Francisco, California 94143, USA. Phone: (415) 502-7395; Fax: (415) 502-8512; E-mail: zamvil@ ucsf.neuroimmunol.org.

Olaf Stüve, Sawsan Youssef, and Martin S. Weber contributed equally to this work.

Scott S. Zamvil and Lawrence Steinman are co-senior authors.
1. Sospedra, M., and Martin, R. 2005. Immunology of multiple sclerosis. Annu. Rev. Immunol. 23:683-747.

2. Zamvil, S.S., and Steinman, L. 2003. Diverse targets for intervention during inflammatory and neurodegenerative phases of multiple sclerosis. Neuron. 38:685-688.

3. Steinman, L., and Zamvil, S.S. 2005. Virtues and pitfalls of EAE for the development of therapies for multiple sclerosis. Trends Immunol. 26:565-571.

4. The IFNB Multiple Sclersosis Study Group. 1993. Interferon beta-1b is effective in relapsing-remitting multiple sclerosis. I. Clinical results of a multicenter, randomized, double-blind, placebo-controlled trial. Neurology. 43:655-661.

5. Johnson, K.P., et al. 1995. Copolymer 1 reduces relapse rate and improves disability in relapsingremitting multiple sclerosis: results of a phase III multicenter, double-blind, placebo-controlled trial. Neurology. 45:1268-1276.

6. Hartung, H.P., et al. 2002. Mitoxantrone in progressive multiple sclerosis: a placebo-controlled, double-blind, randomised, multicentre trial. Lancet. 360:2018-2025.

7. Filippini, G., et al. 2003. Interferons in relapsing remitting multiple sclerosis: a systematic review. Lancet. 361:545-552.

8. Munari, L.M., and Filippini, G. 2004. Lack of evidence for use of glatiramer acetate in multiple sclerosis. Lancet Neurol. 3:641.

9. Soos, J.M., et al. 2002. Cutting edge: oral type I IFN-tau promotes a Th2 bias and enhances suppression of autoimmune encephalomyelitis by oral glatiramer acetate. J. Immunol. 169:2231-2235.

10. Teitelbaum, D., Aharoni, R., Arnon, R., and Sela, M. 1988. Specific inhibition of the T-cell response to myelin basic protein by the synthetic copolymer Cop 1. Proc. Natl. Acad. Sci. U. S. A. 85:9724-9728.

11. Genain, C.P., and Zamvil, S.S. 2000. Specific immunotherapy: one size does not fit all. Nat. Med. 6:1098-1100.

12. Neuhaus, O., et al. 2000. Multiple sclerosis: comparison of copolymer-1- reactive $\mathrm{T}$ cell lines from treated and untreated subjects reveals cytokine shift from Thelper 1 to T helper 2 cells. Proc. Natl. Acad. Sci.U. S. A. 97:7452-7457.

13. Duda, P.W., Schmied, M.C., Cook, S.L., Krieger, J.I., and Hafler, D.A. 2000. Glatiramer acetate (Copax- one) induces degenerate, Th2-polarized immune responses in patients with multiple sclerosis. J. Clin. Invest. 105:967-976.

14. Hussien, Y., Sanna, A., Soderstrom, M., Link, H., and Huang, Y.M. 2001. Glatiramer acetate and IFN-beta act on dendritic cells in multiple sclerosis. J. Neuroimmunol. 121:102-110.

15. Weber, M.S., et al. 2004. Multiple sclerosis: glatiramer acetate inhibits monocyte reactivity in vitro and in vivo. Brain. 127:1370-1378.

16. Kim, H.J., et al. 2004. Type 2 monocyte and microglia differentiation mediated by glatiramer acetate therapy in patients with multiple sclerosis. J. Immunol. 172:7144-7153.

17. Farina, C., Weber, M.S., Meinl, E., Wekerle, H., and Hohlfeld, R. 2005. Glatiramer acetate in multiple sclerosis: update on potential mechanisms of action. Lancet Neurol. 4:567-575.

18. Giuliani, F., et al. 2005. Additive effect of the combination of glatiramer acetate and minocycline in a model of MS. J. Neuroimmunol. 158:213-221.

19. Kwak, B., Mulhaupt, F., Myit, S., and Mach, F. 2000. Statins as a newly recognized type of immunomodulator. Nat. Med. 6:1399-1402.

20. Neuhaus, O., et al. 2002. Statins as immunomodulators: comparison with interferon-beta $1 \mathrm{~b}$ in MS. Neurology. 59:990-997.

21. Zamvil, S.S., and Steinman, L. 2002. Cholesterollowering statins possess anti-inflammatory activity that might be useful for treatment of MS. Neurology. 59:970-971.

22. Vollmer, T., et al. 2004. Oral simvastatin treatment in relapsing-remitting multiple sclerosis. Lancet. 363:1607-1608.

23. McCarey, D.W., et al. 2004. Trial of Atorvastatin in Rheumatoid Arthritis (TARA): double-blind, randomised placebo-controlled trial. Lancet. 363:2015-2021.

24. Youssef, S., et al. 2002. The HMG-CoA reductase inhibitor, atorvastatin, promotes a Th2 bias and reverses paralysis in central nervous system autoimmune disease. Nature. 420:78-84.

25. Aktas, O., et al. 2003. Treatment of relapsing paralysis in experimental encephalomyelitis by targeting Th1 cells through atorvastatin. J. Exp. Med. 197:725-733.

26. Pahan, K., Sheikh, F.G., Namboodiri, A.M., and
Singh, I. 1997. Lovastatin and phenylacetate inhibit the induction of nitric oxide synthase and cytokines in rat primary astrocytes, microglia, and macrophages. J. Clin. Invest. 100:2671-2679.

27. Greenwood, J., et al. 2003. Lovastatin inhibits brain endothelial cell Rho-mediated lymphocyte migration and attenuates experimental autoimmune encephalomyelitis. FASEB J. 17:905-907.

28. Brod, S.A., Lindsey, J.W., and Wolinsky, J.S. 2000. Combination therapy with glatiramer acetate (copolymer-1) and a type I interferon (IFN-alpha) does not improve experimental autoimmune encephalomyelitis. Ann. Neurol. 47:127-131.

29. Teitelbaum, D., Fridkis-Hareli, M., Arnon, R., and Sela, M. 1996. Copolymer 1 inhibits chronic relapsing experimental allergic encephalomyelitis induced by proteolipid protein (PLP) peptides in mice and interferes with PLP-specific T cell responses. J. Neuroimmunol. 64:209-217.

30. Aharoni, R., Teitelbaum, D., Sela, M., and Arnon, R 1997. Copolymer 1 induces $T$ cells of the $T$ helper type 2 that crossreact with myelin basic protein and suppress experimental autoimmune encephalomyelitis. Proc. Natl. Acad. Sci. U. S. A. 94:10821-10826.

31. Aharoni, R., et al. 2000. Specific Th2 cells accumulate in the central nervous system of mice protected against experimental autoimmune encephalomyelitis by copolymer 1. Proc. Natl. Acad. Sci. U. S. A. 97:11472-11477.

32. Newman, C.B., Palmer, G., Silbershatz, H., and Szarek, M. 2003. Safety of atorvastatin derived from analysis of 44 completed trials in 9,416 patients. Am. J. Cardiol. 92:670-676.

33. Zhang, F.L., and Casey, P.J. 1996. Protein prenylation: molecular mechanisms and functional consequences. Annu. Rev. Biochem. 65:241-269.

34. Jorritsma, P.J., Brogdon, J.L., and Bottomly, K. 2003. Role of TCR-induced extracellular signal-regulated kinase activation in the regulation of early IL-4 expression in naive CD4+ T cells. J. Immunol. 170:2427-2434.

35. Dunn, S.E., et al. 2006. Isoprenoids determine Th1/ Th2 fate in pathogenic T cells, providing a mechanism of modulation of autoimmunity by atorvastatin. J. Exp. Med. 203:401-412.

36. Kappos, L., Kuhle, J., Gass, A., Achtnichts, L., and Radue, E.W. 2004. Alternatives to current disease- 
modifying treatment in MS: what do we need and what can we expect in the future? J. Neurol. 251(Suppl. 5):v57-v64.

37. Lublin, F., et al. 2001. A trial to assess the safety of combining therapy with interferon beta-1a and glatiramer acetate in patients with relapsing MS. Neurology. 56(Suppl. 3):A148.

38. Rudick, R.A., et al. 1998. In vivo effects of interferon beta-1a on immunosuppressive cytokines in multiple sclerosis. Neurology. 50:1294-1300.

39. Ersoy, E., Kus, C.N., Sener, U., Coker, I., and Zorlu, Y. 2005. The effects of interferon-beta on interleukin-10 in multiple sclerosis patients. Eur. J. Neurol. 12:208-211.

40. Lu, H.T., et al. 1995. Interferon (IFN) beta acts downstream of IFN-gamma-induced class II transactivator messenger RNA accumulation to block major histocompatibility complex class II gene expression and requires the 48 -kD DNA-binding protein, ISGF3-gamma. J. Exp. Med. 182:1517-1525.

41. Paty, D.W., and Li, D.K. 1993. Interferon beta-1b is effective in relapsing-remitting multiple sclerosis. II. MRI analysis results of a multicenter, randomized, double-blind, placebo-controlled trial. UBC MS/MRI Study Group and the IFNB Multiple Sclerosis Study Group. Neurology. 43:662-667.

42. Stone, L.A., et al. 1997. Characterization of MRI response to treatment with interferon beta-1b: contrast-enhancing MRI lesion frequency as a primary outcome measure. Neurology. 49:862-869.

43. Leppert, D., Waubant, E., Burk, M.R., Oksenberg, J.R., and Hauser, S.L. 1996. Interferon beta-1b inhibits gelatinase secretion and in vitro migration of human $\mathrm{T}$ cells: a possible mechanism for treatment efficacy in multiple sclerosis. Ann. Neurol. 40:846-852.

44. Stuve, O., et al. 1996. Interferon beta- $1 \mathrm{~b}$ decreases the migration of T lymphocytes in vitro: effects on matrix metalloproteinase-9. Ann. Neurol. 40:853-863.

45. Walters, C.E., et al. 2002. Inhibition of Rho GTPases with protein prenyltransferase inhibitors prevents leukocyte recruitment to the central nervous system and attenuates clinical signs of disease in an animal model of multiple sclerosis. J. Immunol. 168:4087-4094.

46. Birnbaum, G.A. and Altafullah, I. 2005. Combination trial of interferon beta 1a (Rebif) and atorvastatin (Lipitor) in patients with relapsing remitting multiple sclerosis. Neurology. 64(Suppl. 1):A385.

47. Hardardottir, F., Baron, J.L., and Janeway, C.A., Jr. 1995. T cells with two functional antigen-specific receptors. Proc. Natl. Acad. Sci. U. S. A. 92:354-358. 